\section{Spironolactone Therapy in Heart Failure Patients with Chronic Kidney Disease}

P. Kandula: Clin Cardiol 2009: 32 (6):347-348

\section{Author's Reply:}

We have read with great interest the comments made by Kandula et $\mathrm{al}^{1}$ about our article ${ }^{2}$ comparing our results with their retrospective analysis about the rate of hyperkalemia in chronic kidney disease (CKD) patients under spironolactone therapy, during chronic heart failure exacerbations (CHFe).

In our population, more than half of the patients (43 out of 76) on spironolactone therapy had moderate CKD (creatinine clearance $[\mathrm{CrCl}]<60 \mathrm{~mL} / \mathrm{min}$ ) despite a near-normal serum creatinine $(1.31 \pm 0.25 \mathrm{mg} / \mathrm{dL})$; none of our patients had severe $\mathrm{CKD}(\mathrm{CrCl}<30 \mathrm{~mL} / \mathrm{min})$. Spironolactone dose, spironolactone withdrawal rate, hyperkalemia rate, hospitalization due to $\mathrm{CHFe}$ or $\mathrm{CKD}$ decompensation, and death were not different between the 2 groups. We additionally did not observe differences between patients who developed hyperkalemia and patients who did not develop it.

Chronic kidney disease and diabetes are associated with higher rates of hyperkalemia in patients under spironolactone therapy, especially during $\mathrm{CHFe}$ when there may be further renal function deterioration. ${ }^{3}$ Kandula et al reported such high rates (31\%) in their study. Comparing our population, they have more diabetic patients $(63 \%)$, diabetes being the only factor independently associated with hyperkalemia development, probably due to hyporenemic hypoaldosteronism. Importantly, $83 \%$ of their patients where medicated with nonsteroid anti-inflammatory drugs and $25 \%$ were under potassium supplementation, 2 recognized risk factors for hyperkalemia in CKD. As the authors point out, these drugs may have contributed to the hyperkalemia rate. Hyperkalemia could be characteristic of their population, not only due to spironolactone, as they only included patients under spironolactone therapy in their analysis, and did not compare those patients with others that were not taking spironolactone.

We think spironolactone therapy in heart failure patients with moderate $\mathrm{CKD}$ is feasible and may show the same benefits as it does in patients without CKD. Close monitoring of potassium and creatinine is crucial in such patients to avoid serious complications. Creatinine clearance should be estimated in all patients (especially in the elderly and diabetics) and those with severe CKD should not be medicated with spironolactone.

Ricardo Lopes M.D., ${ }^{1}$ Patricia Lourenco, M.D., ${ }^{2}$ Joana Mascarenhas, M.D., ${ }^{2}$ Ana Azevedo, PhD., ${ }^{2}$

Paulo Bettencourt, PhD. ${ }^{2}$

${ }^{1}$ Hospital S. João, Departamento de Cardiologia, Porto, Portugal; ${ }^{2}$ Hospital S. Joao, Departamento De Medicina Interna, Porto, Portugal

\section{References}

1. Kandula P, Sham R. Safety of spironolactone use in ambulatory heart failure patients. Clin Cardiol. 2009;32 (6):347-348.

2. Lopes RJ, Lourenço AP, Mascarenhas J, Azevedo A, Bettencourt P. Safety of spironolactone use in ambulatory heart failure patients. Clin Cardiol.. 2008;31(11):509-513.

3. Wencker D. Acute cardio-renal syndrome: progression from congestive heart failure to congestive kidney failure. Curr Heart Fail Rep. 2007;4(3):134-138. 